\title{
RHO Mutations (p.W126L and p.A346P) in Two Japanese Families with Autosomal Dominant Retinitis Pigmentosa
}

\author{
Satoshi Katagiri, ${ }^{1,2}$ Takaaki Hayashi, ${ }^{2}$ Masakazu Akahori, ${ }^{1}$ Takeshi Itabashi, ${ }^{3}$ \\ Jo Nishino, ${ }^{4}$ Kazutoshi Yoshitake, ${ }^{4}$ Masaaki Furuno, ${ }^{5}$ Kazuho Ikeo, ${ }^{4}$ Tetsuji Okada, ${ }^{3}$ \\ Hiroshi Tsuneoka, ${ }^{2}$ and Takeshi Iwata ${ }^{1}$ \\ ${ }^{1}$ Division of Molecular and Cellular Biology, National Institute of Sensory Organs, National Hospital Organization \\ Tokyo Medical Center, 2-5-1 Higashigaoka, Meguro-ku, Tokyo 152-8902, Japan \\ ${ }^{2}$ Department of Ophthalmology, The Jikei University School of Medicine, 3-25-8 Nishi-shimbashi, Minato-ku, Tokyo 105-8461, Japan \\ ${ }^{3}$ Department of Life Science, Gakushuin University, 1-5-1 Mejiro, Toshima-ku, Tokyo 171-8588, Japan \\ ${ }^{4}$ Laboratory of DNA Data Analysis, National Institute of Genetics, 1111 Yata Mishima, Shizuoka 411-8540, Japan \\ ${ }^{5}$ RIKEN Center for Life Science Technologies, Division of Genomic Technologies, Life Science Accelerator Technology Group, \\ Transcriptome Technology Team, 1-7-22 Suehiro-cho, Tsurumi-ku, Yokohama, Kanagawa 230-0045, Japan \\ Correspondence should be addressed to Takaaki Hayashi; taka@jikei.ac.jp
}

Received 26 July 2014; Accepted 20 October 2014; Published 16 November 2014

Academic Editor: Toshihide Kurihara

Copyright (c) 2014 Satoshi Katagiri et al. This is an open access article distributed under the Creative Commons Attribution License, which permits unrestricted use, distribution, and reproduction in any medium, provided the original work is properly cited.

Purpose. To investigate genetic and clinical features of patients with rhodopsin $(R H O)$ mutations in two Japanese families with autosomal dominant retinitis pigmentosa (adRP). Methods. Whole-exome sequence analysis was performed in ten adRP families. Identified RHO mutations for the cosegregation analysis were confirmed by Sanger sequencing. Ophthalmic examinations were performed to evaluate the RP phenotypes. The impact of the RHO mutation on the rhodopsin conformation was examined by molecular modeling analysis. Results. In two adRP families, we identified two RHO mutations (c.377G>T (p.W126L) and c.1036G>C (p.A346P)), one of which was novel. Complete cosegregation was confirmed for each mutation exhibiting the RP phenotype in both families. Molecular modeling predicted that the novel mutation (p.W126L) might impair rhodopsin function by affecting its conformational transition in the light-adapted form. Clinical phenotypes showed that patients with p.W126L exhibited sector RP, whereas patients with p.A346P exhibited classic RP. Conclusions. Our findings demonstrated that the novel mutation (p.W126L) may be associated with the phenotype of sector RP. Identification of RHO mutations is a very useful tool for predicting disease severity and providing precise genetic counseling.

\section{Introduction}

Retinitis pigmentosa (RP) is a heterogeneous group of inherited retinal disorders characterized by night blindness, constricted visual fields, abnormal color vision, and retinal degeneration. The prevalence of RP is approximately 1 per 4000 persons, with more than 1 million affected individuals existing worldwide [1]. RP patients show various inheritance patterns including autosomal recessive, autosomal dominant, $\mathrm{X}$-linked, mitochondrial [2], and digenic [3] inheritance.

Autosomal dominant RP (adRP) makes up 30 to $40 \%$ of the overall RP cases, while mutations in the rhodopsin $(\mathrm{RHO})$ gene are responsible for about $25 \%$ of adRP cases found in Caucasians [1]. The RHO gene has been mapped to the long arm of chromosome 3 (3p21-24) and encodes 348 amino acids [4]. In 1990, the RHO gene was first described in the literature as being the causative gene for adRP $[5,6]$. While rhodopsin is a typical seven transmembrane G-protein-coupled receptor, a photon and not a molecular ligand is responsible for initiating the rhodopsin phototransduction cascade. When rhodopsin absorbs the photon, retinal chromophore (11-cis-retinal) changes to all-trans-retinal. The conformational changes that occur in rhodopsin result in the hyperpolarization of the rod cells, which play an important role in vision [7]. 
Family 1 (JU\#0678-062JIKEI)

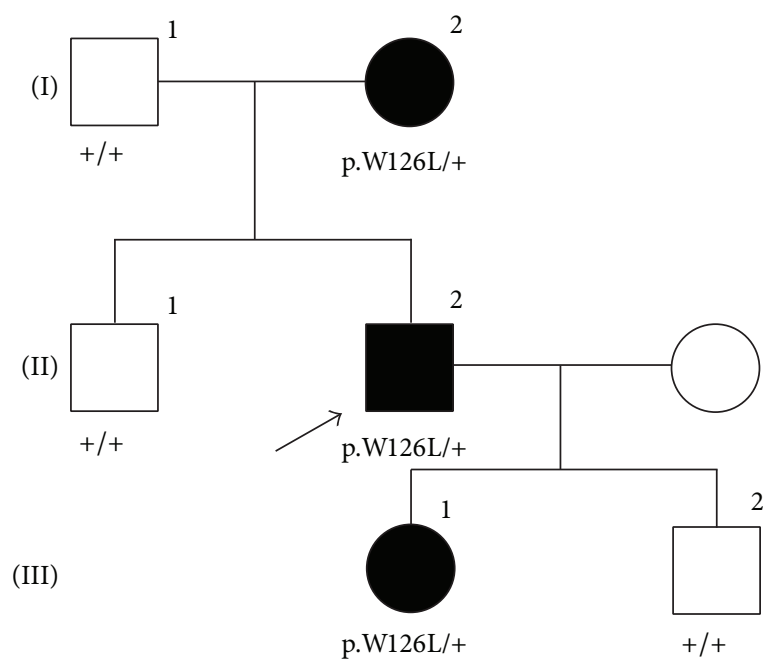

(a)
(I)

Family 2 (JU\#0575-037JIKEI)

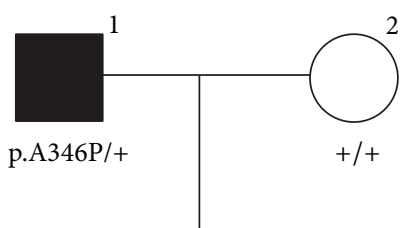

(II)

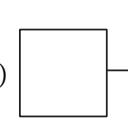

(III)

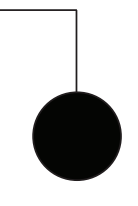

(b)

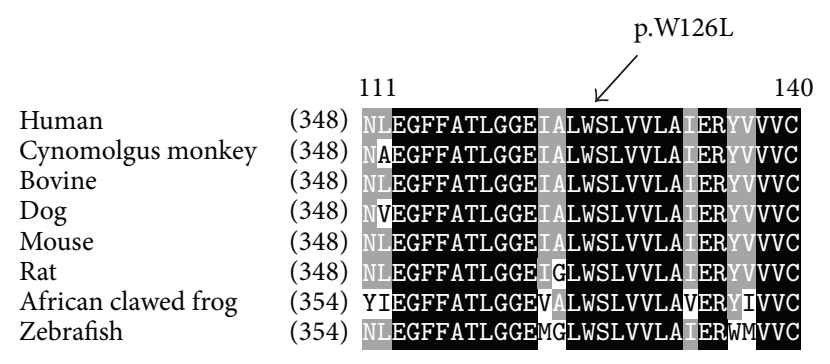

(c)

FIGURE 1: Pedigrees of the two Japanese families with RP and amino acid sequence alignment of the rhodopsin in different vertebrate species. (a) and (b) The solid squares (male) and circles (female) represent the affected individuals. The proband of each family is indicated by the arrows. (c) The tryptophan residue at position 126 is highly conserved. The conserved amino acids between the different species are shown in the black boxes. The less and least conserved amino acids are highlighted in the gray or white boxes, respectively.

Dominant (or heterozygous) $R H O$ mutations have been reported to show two different RP phenotypes, classic RP and sector RP [8-12]. Classic RP is a typical form of RP that is characterized by early-onset and diffuse/generalized retinal dysfunction, whereas sector RP is characterized by adultonset and regionalized/sectorial retinal dysfunction [13-15]. Sector RP, as originally described by Bietti in 1937 [16], is characterized by retinal degeneration that is limited to one or two quadrants of the fundus and slowly progression compared with classic RP [13-15].

The frequency of the RHO mutations in adRP differs between ethnic groups. For example, there is a much lower frequency of RHO mutations in Japanese, Chinese, and Korean populations compared with European populations [17-19]. Saga et al. found RHO mutations in $1 / 13(7.7 \%)$ adRP Japanese patients [20], whereas a separate study found that $43 / 150$ (29\%) adRP patients in North America had the RHO mutations [21]. Thus, RHO mutations have not been considered a major cause of adRP in Japanese patients.

To date, only a small number of RHO mutations have been reported in the Japanese population [20, 22-25]. In our current study, we used a whole-exome sequencing technique and identified two RHO mutations in two Japanese families with
adRP, one (p.W126L) of which was novel. We additionally examined the impact of the p.W126L mutation on rhodopsin conformation by investigating the molecular modeling.

\section{Material and Methods}

The protocol of this study was approved by the Institutional Research Board of The Jikei University School of Medicine and National Hospital Organization Tokyo Medical Center. The protocol adhered to the tenets of the Declaration of Helsinki, and informed consent was obtained from all participants.

2.1. Clinical Studies. Ten unrelated adRP patients from ten Japanese families were recruited for this study. RP diagnosis was based on the visual field, fundus examination, and electroretinogram (ERG) findings. Detailed ophthalmic examinations were conducted in the two families that exhibited the RHO mutations (family 1: JU\#0678-062JIKEI and family 2: JU\#0575-037JIKEI) (Figures 1(a) and 1(b)). These evaluations included decimal best-corrected visual acuity (BCVA), slitlamp, and fundus examinations, optical coherence tomography (OCT) (Cirrus HD-OCT; Carl Zeiss Meditec AG, 
Dublin, CA), and fundus autofluorescence imaging (Spectralis HRA; Heidelberg Engineering, Heidelberg, Germany). Visual fields were assessed with kinetic Goldmann perimetry (GP; Haag Streit, Bern, Switzerland). Full-field ERG was performed according to the protocols of the International Society for Clinical Electrophysiology of Vision. Details of the methods and normal data have been reported previously [26].

\subsection{DNA Preparation and Exome Sequencing Analysis.} After obtaining venous blood samples from ten adRP patients, genomic DNA was extracted. Whole-exome sequencing was performed in all ten adRP patients using a previously described method [27]. The obtained sequence data in the patients were compared with reference human genome sequences (1000 genomes phase 2 reference, hs37d5). Subsequently, we then focused on only the variants that could change the amino acid sequence, such as the nonsynonymous variants, splice acceptor, and donor site variants, and the short insertions and deletions. In the next step, we filtered the remaining variants using the criteria that the frequency of the variant had to be less than $1 \%$ in the databases of the 1000 Genomes project (http://www.1000genomes.org) and the Human Genetic Variation Browser (http://www.genome.med.kyotou.ac.jp/SnpDB/index.html). In the final step, we screened variants residing within the 212 retinal disease-associated genes listed in the RetNet database that was last updated on March 10, 2014 (https://sph.uth.edu/retnet/).

2.3. Sanger Sequencing for RHO Mutations. Sanger sequencing for $R H O$ mutations was conducted in two of the Japanese families, which included probands and other family members (Figures 1(a) and 1(b)). We used two primer pairs: a forward primer (RHO-2F), 5'-CTCCTCAAATCCCTCTCCCACTCCT- $3^{\prime}$, and a reverse primer (RHO-2R), $5^{\prime}$-TCTTCTGCCCTACACCCCTACCCTG-3' for exon 2, and a forward primer (RHO-5F), $5^{\prime}$-CGAACCTCACTAACGTGCCAG- $3^{\prime}$, and a reverse primer (RHO-5R), $5^{\prime}$ GGTCTTGGTGGATGTCCCTTC-3' for exon 5.

2.4. Molecular Modeling and Simulation. The models for molecular dynamics (MD) simulation were generated using two bovine rhodopsin/opsin crystal structures as the templates: the dark-adapted rhodopsin (Protein Data Bank ID: 1U19, chain A) and the ligand-free form opsin (Protein Data Bank ID: 3CAP, chain A). To examine the impact of the p.W126L mutation on protein conformation, 11-cis-retinal was removed from the dark-adapted template. Amino acids in these templates were replaced with the corresponding amino acids from the human sequence, thereby resulting in the wild-type (WT) models. The p.W126L models were generated from the WT models by changing the tryptophan at position 126 to leucine. All amino acid replacements were performed by using the simple mutate function of the Coot software [28]. The membrane environment was an artificially generated palmitoyl-oleoyl-phosphatidyl choline bilayer of approximately 120 molecules with a dimension of $80 \times 80 \AA$. Each model was then merged with the membrane. All of the models we developed were superposed onto each other so that each model was embedded in the artificial membrane at almost identical orientations. The protein-membrane system was then solvated with water containing $150 \mathrm{mM} \mathrm{NaCl}$. All manipulations were performed using Visual Molecular Dynamics version 1.9 [29].

MD simulations were run by the Not (just) Another Molecular Dynamics program [30] through the visual molecular dynamics interface using the following conditions: 1 fs per step, 100,000 steps (100 ps) for minimization, and $1,000,000$ steps ( $1 \mathrm{~ns}$ ), periodic boundary conditions and particle mesh Ewald method [31], cut-off at $10 \AA$, and switching at $9 \AA$. The calculations were carried out under a constant pressure and temperature ensemble at $310 \mathrm{~K}$ and 1 bar. A figure was prepared using the Discovery Studio Visualizer software (Accelrys Inc., San Diego, CA).

\section{Results}

\subsection{Clinical Findings in Family 1 (JU\#0678-062JIKEI)}

3.1.1. Patient II-2. Patient II-2 (a proband) was a 58-year-old man, who was referred to our hospital because of suspicion of RP. BCVA at his first visit to our hospital was 1.5 (with +1.50 diopter (dpt), cylinder (cyl) $-1.25 \mathrm{dpt}$ axial (Ax) $20^{\circ}$ ) in his right eye and 0.7 (with +1.50 dpt., cyl. -0.50 dpt. Ax. $150^{\circ}$ ) in his left eye. No abnormalities were found except for slight senile cataracts in the anterior segments and media of both eyes. Intraocular pressures were within the normal range in both eyes. Fundus examination revealed retinal degeneration around the inferior vascular arcade in his right (Figure 2(a)) and left eyes. The OCT images showed marked thinning of the outer nuclear layer (ONL) and disruption of the inner segment/outer segment (IS/OS) line, except for the foveal region of his right (Figure 2(a)) and left eyes. GP at a previous hospital showed depression of visual fields and an arcuate scotoma at the superior visual field in his right (Figure 2(a)) and left eyes. Full-field ERG showed that there were decreased amplitudes in the rod, standard combined, cone, and $30-\mathrm{Hz}$ flicker responses (Figure 3(a)).

3.1.2. Patient III-1 (a Daughter of Patient II-2). Patient III1 was a 31-year-old woman, who reported night blindness. The clinical findings of patient III-1 were essentially similar to those found for patient II-2. BCVA at her first visit to our hospital was 1.5 (with cyl. $-0.50 \mathrm{dpt}$. Ax. $160^{\circ}$ ) in her right eye and 1.2 (with cyl. $-1.00 \mathrm{dpt}$. Ax. $35^{\circ}$ ) in her left eye. There were no abnormalities found in the anterior segments and media of either eye. Intraocular pressures were within the normal range in both eyes. Fundus examination revealed retinal degeneration in the nasal area of her right (Figure 2(b)) and left eyes. The OCT images showed marked thinning of the ONL and disruption of the IS/OS line except for the foveal region of her right (Figure 2(b)) and left eyes. GP showed several isolated scotomas in her right (Figure 2(b)) and left eyes. Full-field ERG showed that there were decreased amplitudes in the rod, standard combined, cone, and $30-\mathrm{Hz}$ flicker responses (Figure 3(b)). 

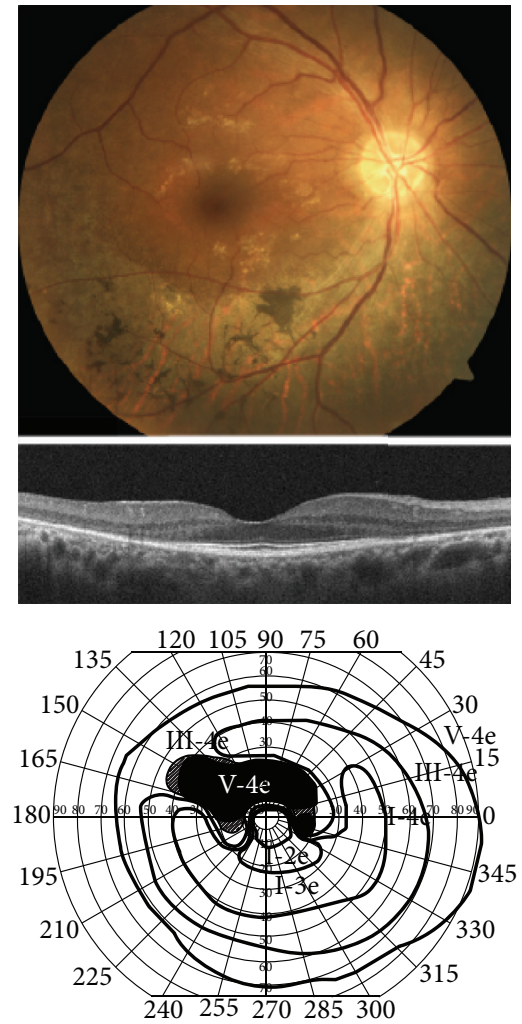

(a)
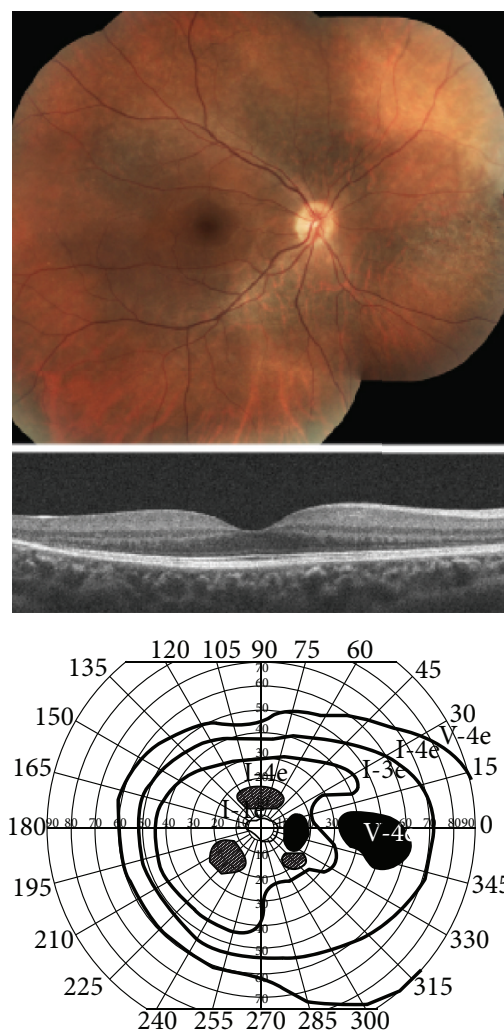

(b)
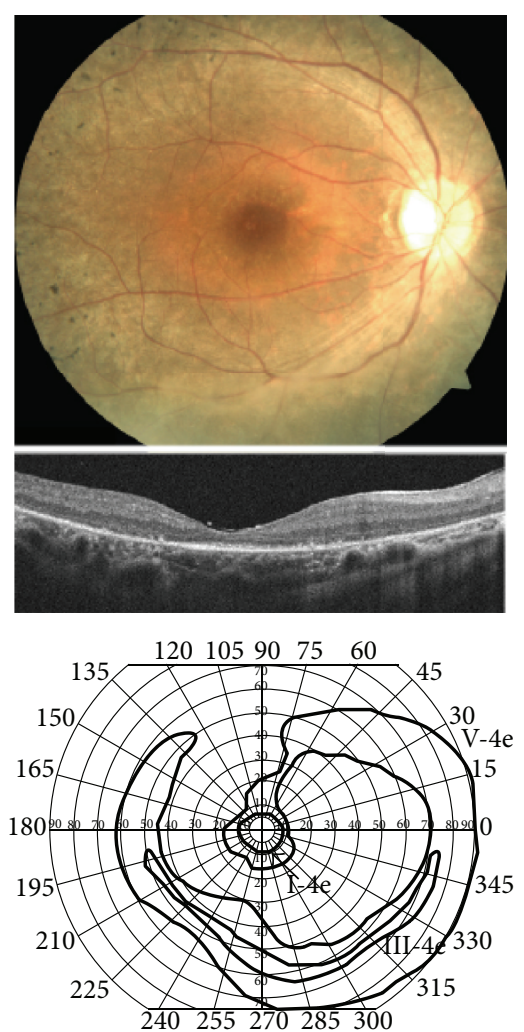

(c)
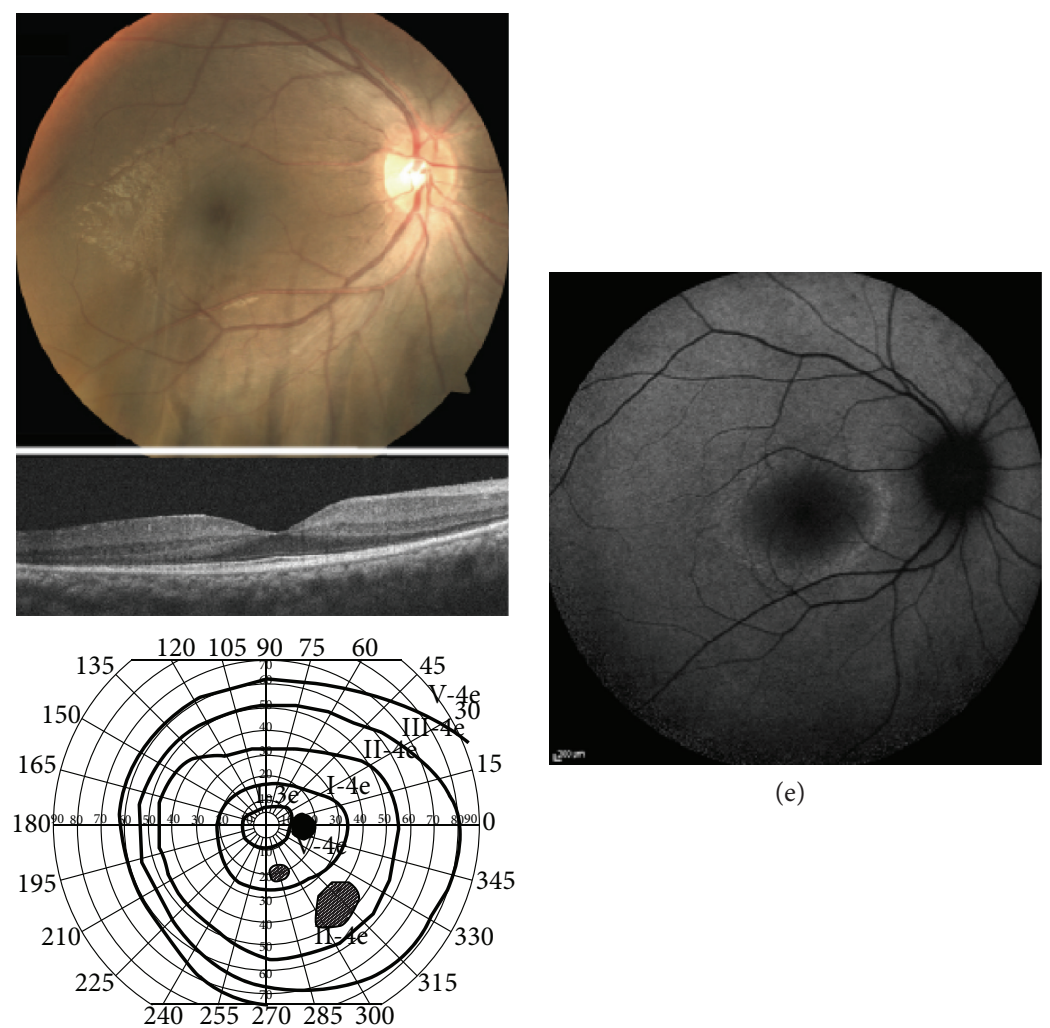

(e)

(d)

FIGURE 2: Fundus photographs, optical coherence tomography (OCT) images, and visual fields with Goldmann kinetic perimetry (GP) for the two Japanese families with retinitis pigmentosa. (a) Fundus photograph, OCT, and GP in the right eye of patient II-2 in family 1 . (b) Fundus photograph, OCT, and GP in the right eye of patient III-1 in family 1. (c) Fundus photograph, OCT, and GP in the right eye of patient II-1 in family 2. (d) Fundus photograph, OCT, and GP in the right eye of patient III-1 in family 2. (e) A fundus autofluorescence image in the right eye of patient III-1 in family 2 . See the Results section for details. 

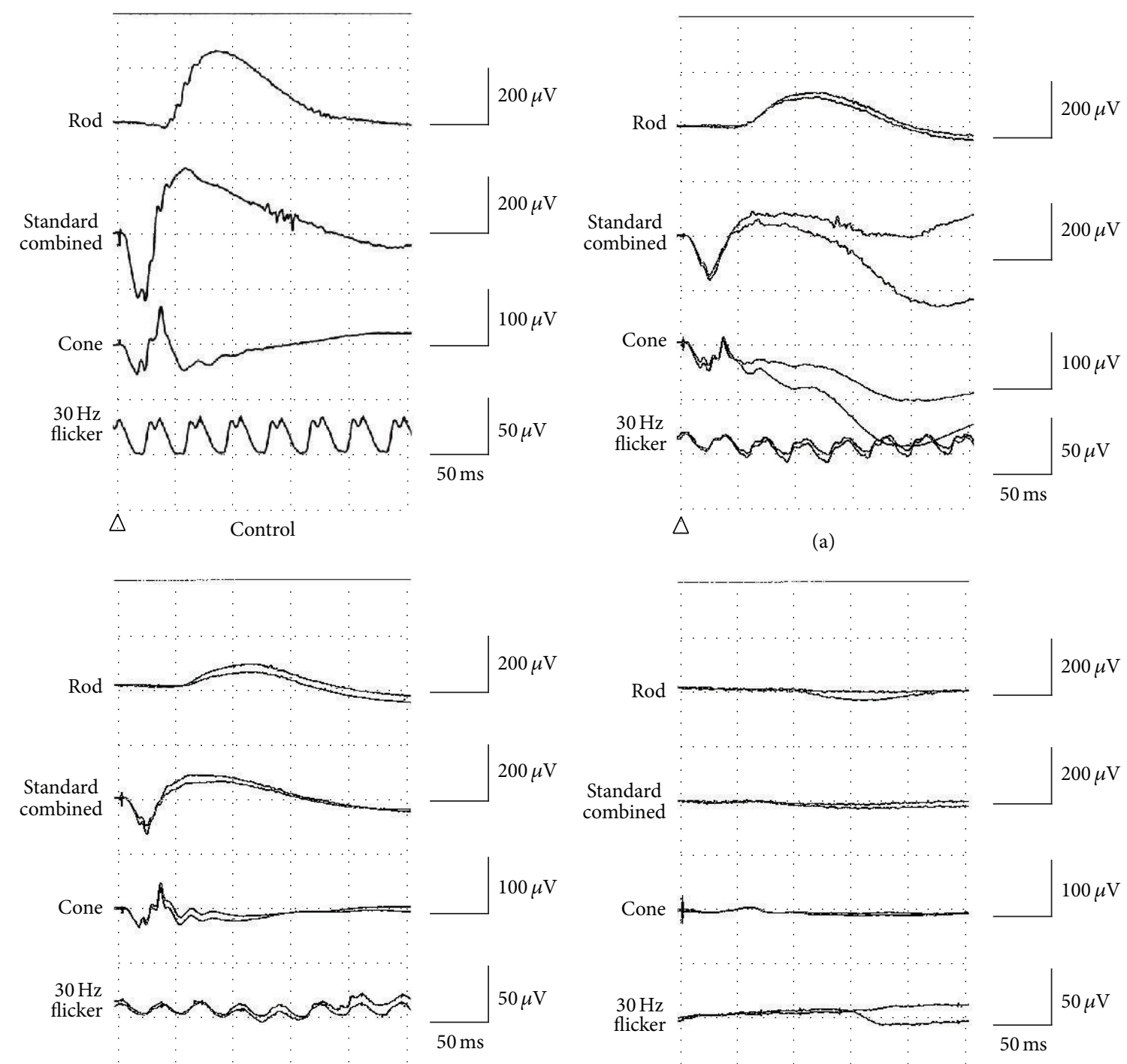

$\triangle$

(b)

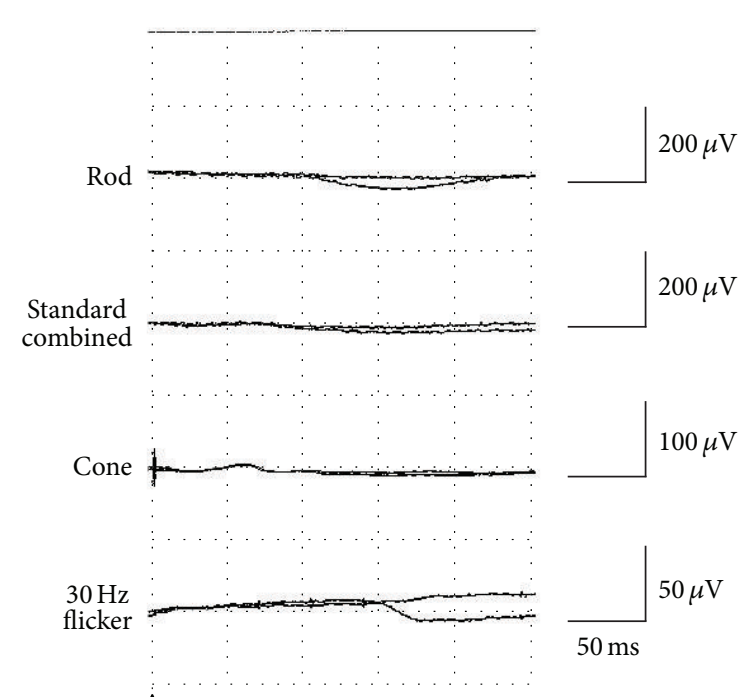

(c)

FIGURE 3: Full-field electroretinograms (ERGs). (a) and (b) ERG of both patient II-2 (a) and patient III-1 (b) in family 1 shows diminished amplitudes of the rod, standard combined, cone, and 30-Hz flicker responses. (c) ERG of patient II-1 in family 2 is nonrecordable for the rod, standard combined and $30-\mathrm{Hz}$ flicker responses.

\subsection{Clinical Findings in Family 2 (JU\#0575-037JIKEI)}

3.2.1. Patient II-1. Patient II-1 (a proband) was a 35 -year-old woman referred to our hospital for assessment of a causative gene for her RP. BCVA was 0.7 (with no correction) in her right eye and 0.6 (with cyl. $-1.75 \mathrm{dpt}$. Ax. $30^{\circ}$ ) in her left eye. There were no abnormalities found in the anterior segments and media of either eye. Intraocular pressures were within the normal range in both eyes. Fundus examination revealed diffuse retinal degeneration and intraretinal pigment deposits with a bone-spicule configuration around the vascular arcade to the periphery of her right (Figure 2(c)) and left eyes. The OCT images showed marked thinning of the ONL and entire disruption of the IS/OS line in her right (Figure 2(c)) and left eyes. GP showed a ring-like defect in her right (Figure 2(c)) and left eyes. Full-field ERG showed that there were no responses in the rod, standard combined, and $30-\mathrm{Hz}$ flicker ERG (Figure 3(c)).

3.2.2. Patient III-1 (a Son of Patient II-1). Patient III-1 was a 14 -year-old boy, who was first found to have night blindness at 6 years of age. At the age of 11, a dark-adapted single flash ERG performed at another hospital showed that there were reduced responses in both eyes (data not shown). BCVA was 1.2 (with +6.50 dpt., cyl. -2.00 dpt. Ax. $180^{\circ}$ ) in his right eye and 1.0 (with +7.00 dpt., cyl. $-1.50 \mathrm{dpt}$. Ax. $10^{\circ}$ ) in his left eye. Fundus examination revealed retinal degeneration of the inferotemporal areas in his right (Figure $2(\mathrm{~d})$ ) and left eyes. The OCT images showed that there was marked thinning of the ONL and disruption of the IS/OS line, except 
TABLE 1: The rare variants found in the two Japanese families with $R H O$ mutations, focusing on 212 retinal disease-causing genes registered in the RetNet database (https://sph.uth.edu/retnet/).

\begin{tabular}{|c|c|c|c|c|c|c|c|c|}
\hline \multicolumn{2}{|c|}{ JU0678-062JIKEI } & \multirow{2}{*}{ Gene } & \multirow{2}{*}{ Gene Bank ID } & \multirow{2}{*}{ Exon } & \multirow{2}{*}{$\begin{array}{c}\text { Nucleotide } \\
\text { change }\end{array}$} & \multirow{2}{*}{ AA change } & \multirow{2}{*}{ State } & \multirow{2}{*}{ SNP ID } \\
\hline Chrom & Position & & & & & & & \\
\hline 2 & 202498104 & TMEM237 & NM_001044385 & 5 & c. $325 \mathrm{C}>\mathrm{T}$ & p.R109X & Hetero & \\
\hline 3 & 129249734 & RHO & NM_000539 & 2 & c. $377 \mathrm{G}>\mathrm{T}$ & p.W126L & Hetero & \\
\hline 4 & 6290790 & WFS1 & NM_006005 & 4 & c. $392 \mathrm{~T}>\mathrm{G}$ & p.V131G & Hetero & \\
\hline 4 & 6302786 & $W F S 1$ & NM_006005 & 8 & c. $1264 \mathrm{G}>\mathrm{T}$ & p.A422S & Hetero & \\
\hline 7 & 33427676 & BBS9 & NM_198428 & 19 & c. $2035 \mathrm{C}>\mathrm{T}$ & p.R679W & Hetero & \\
\hline 8 & 10480476 & $R P 1 L 1$ & NM_178857 & 2 & c. $236 \mathrm{G}>\mathrm{A}$ & p.R79H & Hetero & \\
\hline 14 & 21792816 & RPGRIP1 & NM_020366 & 14 & c. $1802 \mathrm{C}>\mathrm{T}$ & p.S601L & Hetero & rs3748360 \\
\hline 16 & 49670817 & ZNF423 & NM_015069 & 4 & c. $2243 \_2245 \mathrm{del}$ & p.748_749del & Hetero & \\
\hline \multicolumn{2}{|c|}{ JU0575-037JIKEI } & Gene & Gene Bank ID & Exon & Nucleotide & AA change & State & SNP ID \\
\hline Chrom & Position & Gene & Gene Bank ID & Exon & change & AA change & State & SNP ID \\
\hline 1 & 94476477 & $A B C A 4$ & NM_000350 & 40 & c. $5593 \mathrm{C}>\mathrm{T}$ & p.H1865Y & Hetero & rs201707267 \\
\hline 2 & 112751865 & MERTK & NM_006343 & 9 & c. $1334 \mathrm{G}>\mathrm{A}$ & p.R445Q & Hetero & rs202242962 \\
\hline 3 & 129252550 & RHO & NM_000539 & 5 & c. $1036 \mathrm{G}>\mathrm{C}$ & p.A346P & Hetero & \\
\hline 11 & 17531103 & USH1C & NM_153676 & 18 & c. $1813 \mathrm{~A}>\mathrm{C}$ & p.I605L & Hetero & \\
\hline 16 & 16291933 & ABCC6 & NM_001171 & 10 & c. $1283 \mathrm{~A}>\mathrm{G}$ & p.N428S & Hetero & rs201880691 \\
\hline
\end{tabular}

Chrom = choromosome, $\mathrm{AA}=$ amino acid, Homo = homozygous, and Hetero = heterozygous .

for the foveal region of his right (Figure 2(d)) and left eyes. GP showed constriction of visual fields with small scotomas in his right (Figure 2(d)) and left eyes. A fundus autofluorescence image revealed a perifoveal hyperautofluorescent ring in his right (Figure 2(e)) and left eyes. He was diagnosed as an early stage of RP.

3.3. Molecular Genetic Findings. The obtained sequence data were analyzed in accordance with the filtering steps discussed in the Material and Methods section. Table 1 summarizes the remaining rare variants that were examined in families 1 and 2. The analysis focused on the 212 retinal disease-causing genes that were listed in the RetNet database. The two RHO mutations revealed in the data, c.377G > T, p.W126L in exon 2 and c.1036G $>$ C, p.A346P in exon 5, were found in the adRP patients of families 1 and 2, respectively. With the exception of these RHO mutations, there were no other mutations found in the obtained sequence data for both families 1 and 2 that fulfilled the RP phenotype and autosomal dominant inheritance pattern conditions. The p.W126L mutation has not been previously reported in the literature and is not found in the dbSNP (http://www.ncbi.nlm.nih.gov/SNP/), 1000 Genomes database, or the HGMD (http://www.hgmd.org). On the other hand, the p.A346P mutation was previously reported to be the cause of adRP in one Spanish family [34]. We used in silico bioinformatics tools to investigate the impact of the p.W126L mutation on the RHO function. Results of the PolyPhen-2 program generated a score of 0.999 (probably damaging), which was close to the maximum value of 1.00 , while the SIFT program generated a score of 0 (damaging).

For the cosegregation analysis, we investigated whether six of the family 1 members and four of the family 2 members had either the p.W126L or the p.A346P mutation. Our results revealed there was complete cosegregation of each mutation with the RP phenotype in each family (Figures $1(a)$ and 1(b)).

3.4. Molecular Modeling and Simulation. By using energy minimization and MD simulations, we investigated the possible effects of the p.W126L mutation on the structure of the human opsin moiety that was represented by either the dark-adapted like (high affinity for 11-cis-retinal) or the light-adapted like (high affinity for all-trans-retinal) crystal structures. Although the simulation time was limited, all four models examined approached similar levels of equilibria. Because the mutation site W126 is located in the third transmembrane helix (TM3), we analyzed differences in the TM3 backbone and in the nearby helices. In the dark-adapted template, the extracellular and central regions of TM3 did not appear to move significantly during the simulation regardless of the type of side chain (W or L) at position 126 (Figures 4(a) and $4(\mathrm{~b})$ ). This result corroborates the previous observation that the p.W126L mutation affected the 11-cis-retinal binding only marginally in COS-1 cells [35]. Interestingly, in the lightadapted like template, the TM3 helix within the heptahelical bundle moved differently in the WT and the p.W126L mutant models during the 1 ns simulation (Figures $4(\mathrm{c})$ and $4(\mathrm{~d})$ ). While the WT and the p.W126L mutant models exhibited only marginal changes in the TM3 during the first 300 ps of simulation (data not shown), this helix progressively tilted only in the p.W126L mutant during the subsequent $1 \mathrm{~ns}$ of simulation (Figure $4(\mathrm{~d})$ ). Persistence of this tilting during longer periods may lead to a decrease in the distance between the cytoplasmic ends of TM3 and TM6 and may affect the signal-transduction properties of the protein. The residue L125, which is next to W126 in bovine rhodopsin, is in contact with a highly conserved phenylalanine in the middle 


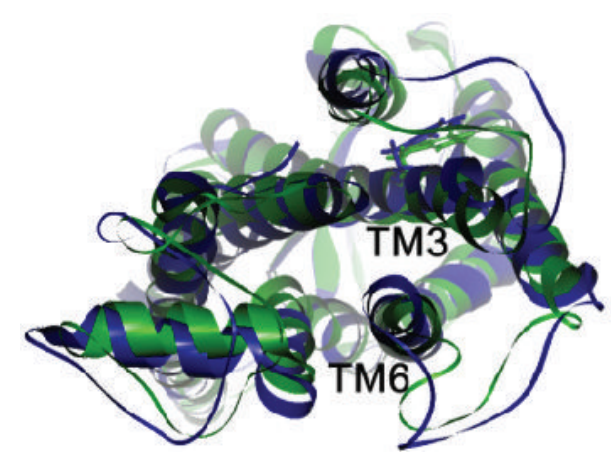

(a)

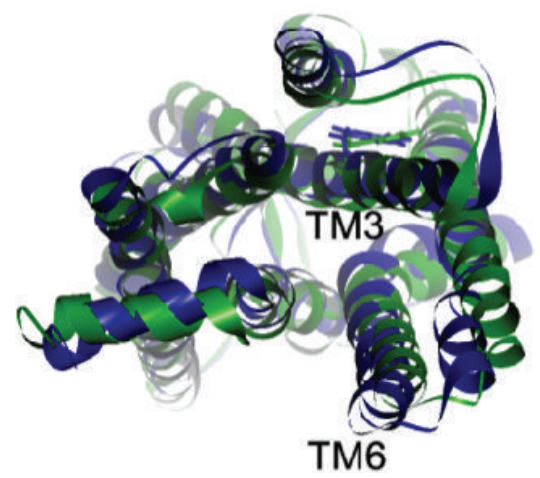

(c)

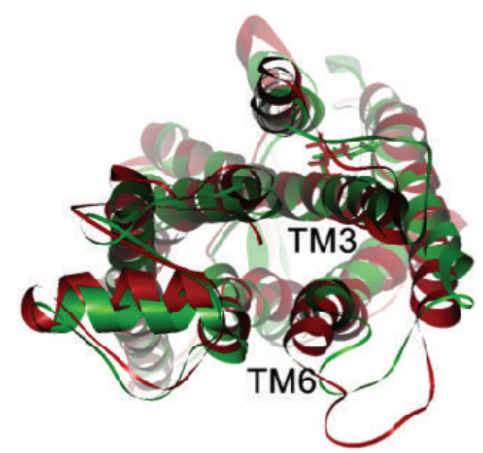

(b)

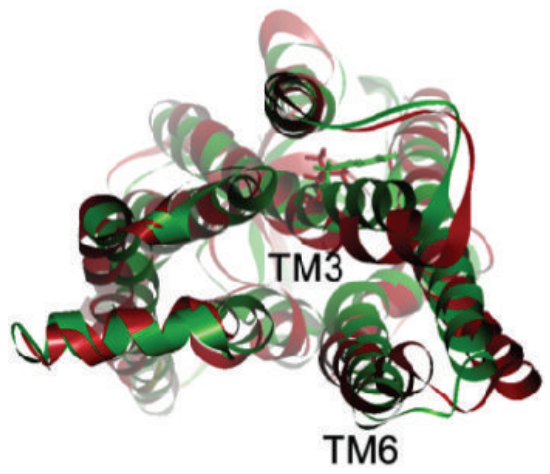

(d)

FIGURE 4: Projected view from the cytoplasmic side of the backbone before and after the 1 ns molecular dynamics (MD) simulations. The side chain of the position 126 in each model is shown by the bold sticks in the corresponding colors. (a) Dark-adapted like models. Blue: wild-type after 1 ns MD; green: wild-type before MD. (b) Dark-adapted like models. Red: W126L after 1 ns MD; green: W126L before MD. (c) Light-adapted like models. Blue: wild-type after $1 \mathrm{~ns}$ MD; green: wild-type before MD. (d) Light-adapted like models. Red: W126L after 1 ns MD; green: W126L before MD.

of the TM6 at residue 261. These side-chain interactions may therefore be critical for the ligand-induced activation of the rhodopsin-like G-protein-coupled receptors, similar to that which has been proposed for the $\beta 2$-adrenergic receptor [36].

\section{Discussion}

In this study, we identified two RHO mutations (p.W126L and p.A346P) as disease causes by using whole-exome sequencing in two Japanese families with adRP. We additionally used molecular modeling to analyze the impact of the novel mutation (p.W126L) on the protein structure and function and then evaluated the genotype-phenotype correlations among Japanese RP patients with heterozygous $R H O$ mutations.

For the cosegregation analysis, further validation by Sanger sequencing in other family members demonstrated there was complete cosegregation of each mutation with the RP phenotype (Figures 1(a) and 1(b)). For the novel p.W126L mutation, the tryptophan residue at the position 126 is located in the TM3 and is highly conserved among orthologs in vertebrates (Figure 1(c)). A biochemical experiment using reconstituted bovine rhodopsin has shown that an analog of 11-cis-retinal is cross-linked to the W126 residue [37]. Our in silico study, which used the PolyPhen-2 and SIFT programs, predicted that the p.W126L mutation would cause severe damage to the rhodopsin. In addition, the results of our protein modeling and simulations (Figure 4) suggest that the p.W126L mutation will likely affect the side-chain interaction between TM3 and TM6 in the light-adapted form but will not affect the interaction in the dark-adapted form. These molecular modeling and simulation findings suggest that the p.W126L mutation may impair rhodopsin function by affecting its conformational transition in the light-adapted form. This interpretation is in line with previous studies that have shown that p.W126L of bovine rhodopsin is less potent during G-protein activation [35]. Thus, these results predict that the p.W126L mutation can affect the RHO function, especially in the light-adapted form, thereby leading to the phenotype of RP. On the other hand, p.A346P has previously been reported to be an adRP-causing mutation in one Spanish family [34]. Both our cosegregation data for each family and the molecular modeling confirm that p.W126L is a diseasecausing mutation.

With regard to the phenotypes of our patients, the p.W126L (family 1) and p.A346P (family 2) mutations are likely to be associated with sector RP and classic RP, respectively. However, it should be noted that patient III-1 (family 2) was not diagnosed with either classic or sector $\mathrm{RP}$, as patient III-1 exhibited an early stage of RP. The 
TABLE 2: Clinical summary of Japanese patients with autosomal dominant retinitis pigmentosa with heterozygous $R H O$ mutations.

\begin{tabular}{|c|c|c|c|c|c|c|c|c|c|}
\hline \multirow[b]{2}{*}{ Patient, gender } & \multirow[b]{2}{*}{ Type of adRP } & \multirow{2}{*}{$\begin{array}{c}\text { Age at } \\
\text { examination }\end{array}$} & \multirow[b]{2}{*}{ Mutation } & \multicolumn{2}{|c|}{ BCVA } & \multicolumn{2}{|c|}{ Electroretinograms (ERGs) } & \multirow[b]{2}{*}{ Reference } & \multirow[b]{2}{*}{ Notes } \\
\hline & & & & $\mathrm{R}$ & $\mathrm{L}$ & $\begin{array}{c}\text { Flash (rod } \\
\text { plus cone) } \\
\text { ERG }\end{array}$ & $\begin{array}{l}\text { Full-field } \\
\text { ERG }\end{array}$ & & \\
\hline Case $1, \mathrm{M}$ & Classic & 44 & p.P347L & 0.5 & 0.66 & NR & $\begin{array}{l}\mathrm{NR} \text { in } 30-\mathrm{Hz} \\
\text { flicker }\end{array}$ & {$[23,32]$} & Cataract \\
\hline Case 2, F & Classic & 20 & p.P347L & 1.0 & 1.0 & NR & $\begin{array}{l}\mathrm{NR} \text { in } 30-\mathrm{Hz} \\
\text { flicker }\end{array}$ & {$[23,32]$} & \\
\hline Case 3, F & ND & 11 & p.P347L & 1.0 & 1.0 & Reduced & $\begin{array}{l}\text { Reduced in } \\
30-\mathrm{Hz} \text { flicker }\end{array}$ & {$[23,32]$} & \\
\hline Case $4, \mathrm{~F}$ & Classic & 75 & p.P347L & LP & LP & NR & $\begin{array}{c}\text { NR in } 30-\mathrm{Hz} \\
\text { flicker }\end{array}$ & {$[23,32]$} & $\begin{array}{c}\text { Severe } \\
\text { cataract }\end{array}$ \\
\hline III-6, M & Sector & 49 & p.T17M & 0.2 & 0.2 & ND & $\begin{array}{l}\text { Reduced in } \\
\text { both rods } \\
\text { and cones }\end{array}$ & {$[23,33]$} & $\begin{array}{l}\text { B-CME, } \\
\text { L-CNV }\end{array}$ \\
\hline Proband, F & Classic & 39 & p.E181K & 0.1 & 0.1 & ND & $\begin{array}{l}\text { NR in rods, } \\
\text { reduced in } \\
\text { cones }\end{array}$ & {$[20]$} & B-CME \\
\hline III-5, F & Sector & 52 & p.N15S & ND & $\mathrm{ND}$ & ND & ND & {$[24]$} & \\
\hline II-2, M & Sector & 66 & p.G106R & 0.04 & 0.5 & ND & $\begin{array}{l}\text { Reduced in } \\
\text { both rods } \\
\text { and cones }\end{array}$ & {$[25]$} & R-CME \\
\hline III-1, F & Sector & 44 & p.G106R & 1.2 & 1.2 & ND & $\begin{array}{l}\text { Reduced in } \\
\text { rods, normal } \\
\text { in cones }\end{array}$ & {$[25]$} & \\
\hline III-2, F & Sector & 40 & p.G106R & 1.2 & 1.2 & ND & $\begin{array}{l}\text { Reduced in } \\
\text { rods, normal } \\
\text { in cones }\end{array}$ & {$[25]$} & \\
\hline II-2 (FN.1), M & Sector & 58 & p.W126L & 1.5 & 0.7 & ND & $\begin{array}{l}\text { Reduced in } \\
\text { both rods } \\
\text { and cones }\end{array}$ & Current study & \\
\hline III-1 (FN.1), F & Sector & 31 & p.W126L & 1.5 & 1.2 & ND & $\begin{array}{l}\text { Reduced in } \\
\text { both rods } \\
\text { and cones }\end{array}$ & Current study & \\
\hline II-1 (FN.2), F & Classic & 35 & p.A346P & 0.7 & 0.6 & ND & $\begin{array}{l}\text { NR in both } \\
\text { rods and } \\
\text { cones }\end{array}$ & Current study & \\
\hline III-1 (FN.2), M & $\mathrm{ND}$ & 14 & p.A346P & 1.2 & 1.5 & Reduced & ND & Current study & \\
\hline
\end{tabular}

$\mathrm{BCVA}=$ decimal best-corrected visual acuity; $\mathrm{R}=$ right eye; $\mathrm{L}=$ left eye; $\mathrm{B}=$ both eyes; $\mathrm{M}=$ male; $\mathrm{F}=$ female; $\mathrm{FN}=$ family number; $\mathrm{ND}=$ not described or not done; $\mathrm{NR}=$ nonrecordable; $\mathrm{LP}=$ light perception; $\mathrm{CNV}=$ choroidal neovascularization; and $\mathrm{CME}=$ cystoid macular edema.

phenotypes of patient II-1 (family 2) were similar to those previously reported for a patient of European descent who carried the p.A346P mutation and exhibited classic RP [34]. These findings suggest that the p.A346P mutation might be associated with the phenotype of classic RP.

When trying to diagnose classic or sector RP, it is highly important that one understands the severity and prognosis of $\mathrm{RHO}$-associated adRP. As compared to classic RP, sector $\mathrm{RP}$ is considered to be a less severe disease with subnormal ERG and visual field defects that correspond to the affected sectors $[13,14]$. In fact, it has been reported that sector RP is caused by a number of RHO mutations, including p.T4K [38], p.N15S [39-41], p.T17M [33, 42], p.P23H [10], p.T58R [12], p.N78I [43], and G106R [11, 25, 44]. To the best of our knowledge, there have been nine $R H O$ mutations reported in the Japanese adRP population [20, 22-25, 32, 33, 41], with detailed phenotypes described in five (p.N15S, p.T17M, p.G106R, p.E181K, and p.P347L) out of the nine mutations [20, 23-25, 32, 33, 41]. Among these five RHO mutations, one (p.P347L) exhibited classic RP while the other four (p.N15S, p.T17M, p.G106R, and p.E181K) showed sector RP. Table 2 summarizes the clinical features that were described for the genotype-phenotype correlations of the seven Japanese adRP families (including our families) with the seven $\mathrm{RHO}$ mutations (p.N15S, p.T17M, p.G106R, p.W126L, p.E181K, p.A346P, and p.P347L). Interestingly, patients with five other mutations (p.N15S [39-41], p.T17M [33, 42], p.G106R [11, 25, 44], p.A346P [34], and p.P347L [5, 32, 45]) were found to have phenotypes that were similar to Japanese and other ethnic groups, although the clinical phenotypes for two 
other mutations (p.W126L and p.E181K $[20,21,46])$ could not be sufficiently evaluated $[5,11,20,21,25,32-34,39-$ $42,44-46]$. These findings suggest that the location of each missense mutation is important for the purpose of predicting a diagnosis of either classic or sector RP and that there is a similarity of phenotypes between Japanese and other ethnic group RP patients who have identical RHO mutations. Also Sandberg et al. report the phenotype-genotype correlations between adRP patients with $R H O$ mutations, revealing that patients with mutations altering the intradiscal domain near the N-terminal region (or a low-numbered codon) tended to have better visual function than patients with mutations altering the cytoplasmic domain near the $\mathrm{C}$-terminal region (or a high numbered codon); patients with a mutation altering the transmembrane domain or a mid-numbered codon had intermediate function [47]. Thus, identification of $R H O$ mutations appears to be useful for predicting the severity of the RP phenotypes and providing precise genetic counseling.

In conclusion, we identified two RHO mutations (p.W126L and p.A346P) in two Japanese families with adRP. The p.W126L mutation has not been previously reported in any ethnic groups. The genotype-phenotype correlations indicated that the location of the $R H O$ mutations is likely to determine the phenotype of either classic or sector RP. Identification of $R H O$ mutations is a very useful tool for predicting the disease severity and providing precise genetic counseling.

\section{Conflict of Interests}

The authors declare there are no conflict of interests.

\section{Acknowledgments}

This study was supported by the Grants to Takeshi Iwata from the Ministry of Health, Labor and Welfare of Japan (13803661), to Masakazu Akahori and Takaaki Hayashi from the Ministry of Education, Culture, Sports, Science and Technology of Japan (Grant-in-Aid for Scientific Research C, 25462744 and 25462738), to Takaaki Hayashi from the Vehicle Racing Commemorative Foundation, and to Masaaki Furuno from the research Grant for RIKEN Omics Science Center MEXT. The authors wish to acknowledge RIKEN GeNAS for the sequencing of the exome enriched libraries using the Illumina HiSeq2000.

\section{References}

[1] D. T. Hartong, E. L. Berson, and T. P. Dryja, "Retinitis pigmentosa," The Lancet, vol. 368, no. 9549, pp. 1795-1809, 2006.

[2] F. C. Mansergh, S. Millington-Ward, A. Kennan et al., "Retinitis pigmentosa and progressive sensorineural hearing loss caused by a C12258A mutation in the mitochondrial MTTS2 gene," The American Journal of Human Genetics, vol. 64, no. 4, pp. 971-985, 1999.

[3] K. Kajiwara, E. L. Berson, and T. P. Dryja, "Digenic retinitis pigmentosa due to mutations at the unlinked peripherin/RDS and ROM1 loci," Science, vol. 264, no. 5165, pp. 1604-1608, 1994.
[4] J. Nathans and D. S. Hogness, "Isolation and nucleotide sequence of the gene encoding human rhodopsin," Proceedings of the National Academy of Sciences of the United States of America, vol. 81, no. 15 I, pp. 4851-4855, 1984.

[5] T. P. Dryja, T. L. McGee, L. B. Hahn et al., "Mutations within the rhodopsin gene in patients with autosomal dominant retinitis pigmentosa," The New England Journal of Medicine, vol. 323, no. 19, pp. 1302-1307, 1990.

[6] T. P. Dryja, T. L. McGee, E. Reichel et al., "A point mutation of the rhodopsin gene in one form of retinitis pigmentosa," Nature, vol. 343, no. 6256, pp. 364-366, 1990.

[7] R. E. Stenkamp, D. C. Teller, and K. Palczewski, "Rhodopsin: a structural primer for G-protein coupled receptors," Archiv der Pharmazie, vol. 338, no. 5-6, pp. 209-216, 2005.

[8] E. L. Berson, B. Rosner, M. A. Sandberg, and T. P. Dryja, "Ocular findings in patients with autosomal dominant retinitis pigmentosa and a rhodopsin gene defect (Pro-23-His)," Archives of Ophthalmology, vol. 109, no. 1, pp. 92-101, 1991.

[9] E. L. Berson, B. Rosner, M. A. Sandberg, C. Weigel-DiFranco, and T. P. Dryja, "Ocular findings in patients with autosomal dominant retinitis pigmentosa and rhodopsin, proline-347leucine," The American Journal of Ophthalmology, vol. 111, no. 5, pp. 614-623, 1991.

[10] J. R. Heckenlively, J. A. Rodriguez, and S. P. Daiger, "Autosomal dominant sectoral retinitis pigmentosa. Two families with transversion mutation in codon 23 of rhodopsin," Archives of Ophthalmology, vol. 109, no. 1, pp. 84-91, 1991.

[11] G. A. Fishman, E. M. Stone, L. D. Gilbert, and V. C. Sheffield, "Ocular findings associated with a rhodopsin gene codon 106 mutation: glycine-to-arginine change in autosomal dominant retinitis pigmentosa," Archives of Ophthalmology, vol. 110, no. 5, pp. 646-653, 1992.

[12] C. F. Inglehearn, T. J. Keen, R. Bashir et al., "A completed screen for mutations of the rhodopsin gene in a panel of patients with autosomal dominant retinitis pigmentosa," Human Molecular Genetics, vol. 1, no. 1, pp. 41-45, 1992.

[13] A. E. Krill, D. Archer, and D. Martin, "Sector retinitis pigmentosa," American Journal of Ophthalmology, vol. 69, no. 6, pp. 977-987, 1970.

[14] E. L. Berson and J. Howard, "Temporal aspects of the electroretinogram in sector retinitis pigmentosa," Archives of Ophthalmology, vol. 86, no. 6, pp. 653-665, 1971.

[15] R. W. Massof and D. Finkelstein, "Two forms of autosomal dominant primary retinitis pigmentosa," Documenta Ophthalmologica, vol. 51, no. 4, pp. 289-346, 1981.

[16] G. B. Bietti, "Su alcune forme atipiche o rare di degenerazione retina (degenerazioni tuppetoretiniche e quardi morbosi similari)," Boll Oculist, vol. 16, pp. 1159-1244, 1937.

[17] W. M. Chan, K. Y. Yeung, C. P. Pang et al., "Rhodopsin mutations in Chinese patients with retinitis pigmentosa," The British Journal of Ophthalmology, vol. 85, no. 9, pp. 1046-1048, 2001.

[18] Y. Wada and M. Tamai, "Molecular genetic analysis for Japanese patients with autosomal dominant retinitis pigmentosa," Nippon Ganka Gakkai Zasshi, vol. 107, no. 11, pp. 687-694, 2003.

[19] K. J. Kim, C. Kim, J. Bok et al., "Spectrum of rhodopsin mutations in Korean patients with retinitis pigmentosa," Molecular Vision, vol. 17, pp. 844-853, 2011.

[20] M. Saga, Y. Mashima, K. Akeo, Y. Oguchi, J. Kudoh, and N. Shimizu, "Autosomal dominant retinitis pigmentosa. A mutation in codon 181 (Glu $\rightarrow$ Lys) of the rhodopsin gene in a 
Japanese family," Ophthalmic Genetics, vol. 15, no. 2, pp. 61-67, 1994.

[21] T. P. Dryja, L. B. Hahn, G. S. Cowley, T. L. McGee, and E. L. Berson, "Mutation spectrum of the rhodopsin gene among patients with autosomal dominant retinitis pigmentosa," Proceedings of the National Academy of Sciences of the United States of America, vol. 88, no. 20, pp. 9370-9374, 1991.

[22] Z.-B. Jin, M. Mandai, T. Yokota et al., "Identifying pathogenic genetic background of simplex or multiplex retinitis pigmentosa patients: a large scale mutation screening study," Journal of Medical Genetics, vol. 45, no. 7, pp. 465-472, 2008.

[23] K. Fujiki, Y. Hotta, M. Hayakawa et al., "Point mutations of rhodopsin gene found in Japanese families with autosomal dominant retinitis pigmentosa (ADRP)," The Japanese Journal of Human Genetics, vol. 37, no. 2, pp. 125-132, 1992.

[24] K. Fujiki, Y. Hotta, A. Murakami et al., "Missense mutation of rhodopsin gene codon 15 found in Japanese autosomal dominant retinitis pigmentosa," Japanese Journal of Human Genetics, vol. 40, no. 3, pp. 271-277, 1995.

[25] M. Matsumoto, S. Hayasaka, T. Yamada, and Y. Hayasaka, "Rhodopsin gene codon 106 mutation (Gly-to-Arg) in a Japanese family with autosomal dominant retinitis pigmentosa," Japanese Journal of Ophthalmology, vol. 44, no. 6, pp. 610-614, 2000.

[26] T. Takeuchi, T. Hayashi, M. Bedell, K. Zhang, H. Yamada, and H. Tsuneoka, "A novel haplotype with the R345W mutation in the EFEMP1 gene associated with autosomal dominant drusen in a Japanese family," Investigative Ophthalmology and Visual Science, vol. 51, no. 3, pp. 1643-1650, 2010.

[27] S. Katagiri, K. Yoshitake, M. Akahori et al., "Whole-exome sequencing identifies a novel ALMS1 mutation (p.Q2051X) in two Japanese brothers with Alström syndrome," Molecular Vision, vol. 19, pp. 2393-2406, 2013.

[28] P. Emsley, B. Lohkamp, W. G. Scott, and K. Cowtan, "Features and development of Coot," Acta Crystallographica Section D: Biological Crystallography, vol. 66, part 4, pp. 486-501, 2010.

[29] W. Humphrey, A. Dalke, and K. Schulten, "VMD: visual molecular dynamics," Journal of Molecular Graphics, vol. 14, no. 1, pp. 33-38, 1996.

[30] J. C. Phillips, R. Braun, W. Wang et al., "Scalable molecular dynamics with NAMD," Journal of Computational Chemistry, vol. 26, no. 16, pp. 1781-1802, 2005.

[31] T. Darden, D. York, and L. Pedersen, "Particle mesh Ewald: an $\mathrm{N} \cdot \log (\mathrm{N})$ method for Ewald sums in large systems," The Journal of Chemical Physics, vol. 98, no. 12, pp. 10089-10092, 1993.

[32] T. Shiono, Y. Hotta, M. Noro et al., "Clinical features of Japanese family with autosomal dominant retinitis pigmentosa caused by point mutation in codon 347 of rhodopsin gene," Japanese Journal of Ophthalmology, vol. 36, no. 1, pp. 69-75, 1992.

[33] M. Hayakawa, Y. Hotta, Y. Imai et al., "Clinical features of autosomal dominant retinitis pigmentosa with rhodopsin gene codon 17 mutation and retinal neovascularization in a Japanese patient," American Journal of Ophthalmology, vol. 115, no. 2, pp. 168-173, 1993.

[34] P. M. de Abreu, P. G. Farías, G. S. Paiva, A. M. Almeida, and P. V. Morais, "Persistence of microbial communities including Pseudomonas aeruginosa in a hospital environment: a potential health hazard," BMC Microbiology, vol. 14, article 118, no. 2, pp. 180-181, 1996.

[35] T. A. Nakayama and H. G. Khorana, "Mapping of the amino acids in membrane-embedded helices that interact with the retinal chromophore in bovine rhodopsin," The Journal of Biological Chemistry, vol. 266, no. 7, pp. 4269-4275, 1991.

[36] S. G. F. Rasmussen, H.-J. Choi, J. J. Fung et al., "Structure of a nanobody-stabilized active state of the $\beta_{2}$ adrenoceptor," Nature, vol. 469, no. 7329, pp. 175-180, 2011.

[37] T. A. Nakayama and H. G. Khorana, "Orientation of retinal in bovine rhodopsin determined by cross-linking using a photoactivatable analog of 11-cis-retinal," Journal of Biological Chemistry, vol. 265, no. 26, pp. 15762-15769, 1990.

[38] L. I. van den Born, M. J. van Schooneveld, L. A. M. S. de Jong et al., "Thr4Lys rhodopsin mutation is associated with autosomal dominant retinitis pigmentosa of the cone-rod type in a small Dutch family," Ophthalmic Genetics, vol. 15, no. 2, pp. 51-60, 1994.

[39] H. Kranich, S. Bartkowski, M. J. Denton et al., "Autosomal dominant "sector" retinitis pigmentosa due to a point mutation predicting an Asn-15-Ser substitution of rhodopsin," Human Molecular Genetics, vol. 2, no. 6, pp. 813-814, 1993.

[40] L. J. Sullivan, G. S. Makris, P. Dickinson et al., "A new codon 15 rhodopsin gene mutation in autosomal dominant retinitis pigmentosa is associated with sectorial disease," Archives of Ophthalmology, vol. 111, no. 11, pp. 1512-1517, 1993.

[41] M. Yoshii, A. Murakami, K. Akeo et al., "Visual function in retinitis pigmentosa related to a codon 15 rhodopsin gene mutation," Ophthalmic Research, vol. 30, no. 1, pp. 1-10, 1998.

[42] C. Bell, C. A. Converse, H. M. Hammer, A. Osborne, and N. E. Haites, "Rhodopsin mutations in a Scottish retinitis pigmentosa population, including a novel splice site mutation in intron four," The British Journal of Ophthalmology, vol. 78, no. 12, pp. 933-938, 1994.

[43] D. Rivera-De la Parra, J. Cabral-Macias, M. Matias-Florentino, G. Rodriguez-Ruiz, V. Robredo, and J. C. Zenteno, "Rhodopsin p.N78I dominant mutation causing sectorial retinitis pigmentosa in a pedigree with intrafamilial clinical heterogeneity," Gene, vol. 519, no. 1, pp. 173-176, 2013.

[44] C. Ayuso, C. Reig, B. Garcia-Sandoval et al., "G106R rhodopsin mutation is also present in Spanish ADRP patients," Ophthalmic Genetics, vol. 17, no. 3, pp. 95-101, 1996.

[45] S. Bhattacharya, D. Lester, J. Keen et al., "Retinitis pigmentosa and mutations in rhodopsin," The Lancet, vol. 337, no. 8734, p. $185,1991$.

[46] S. Bunge, H. Wedemann, D. David et al., "Molecular analysis and genetic mapping of the rhodopsin gene in families with autosomal dominant retinitis pigmentosa," Genomics, vol. 17, no. 1, pp. 230-233, 1993.

[47] M. A. Sandberg, C. Weigel-DiFranco, T. P. Dryja, and E. L. Berson, "Clinical expression correlates with location of rhodopsin mutation in dominant retinitis pigmentosa," Investigative Ophthalmology \& Visual Science, vol. 36, no. 9, pp. 19341942, 1995. 


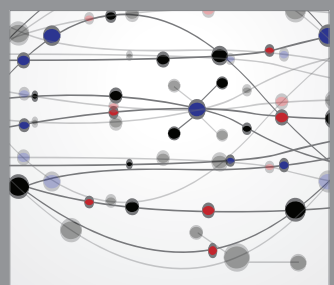

The Scientific World Journal
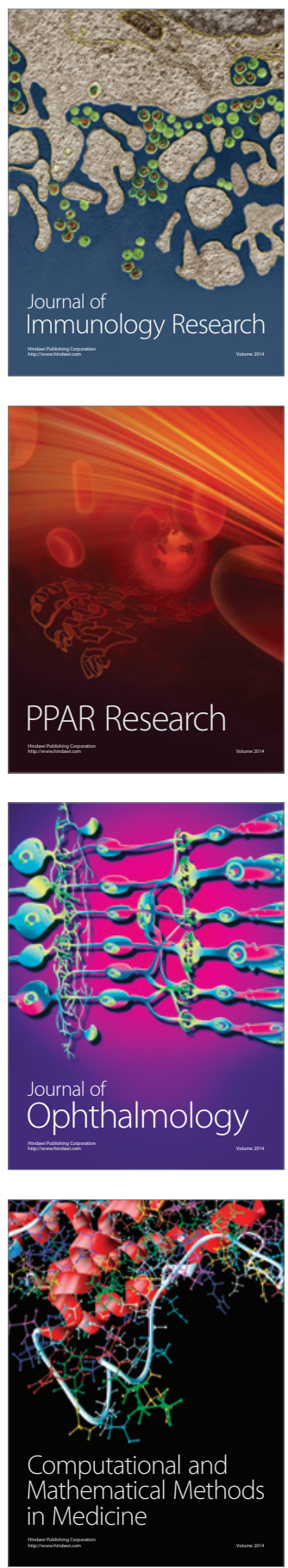

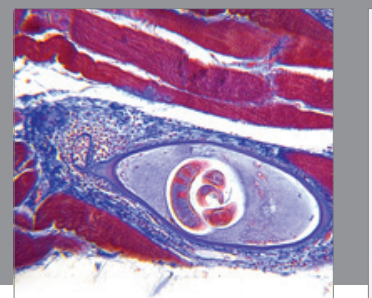

Gastroenterology

Research and Practice
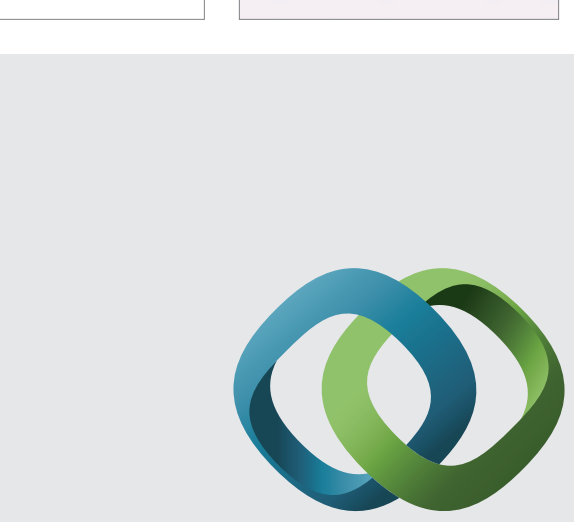

\section{Hindawi}

Submit your manuscripts at

http://www.hindawi.com
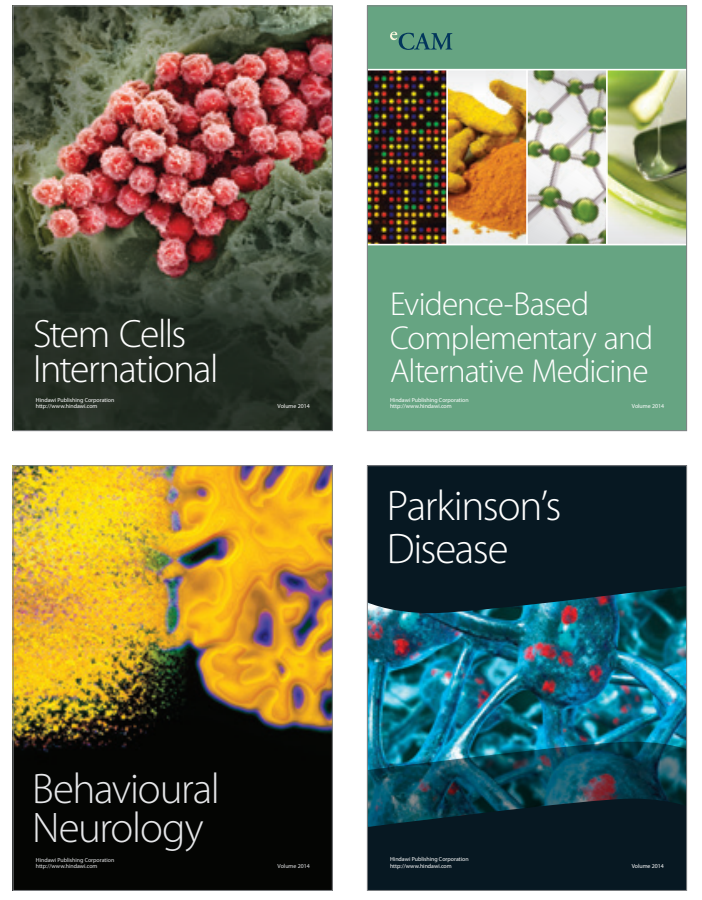
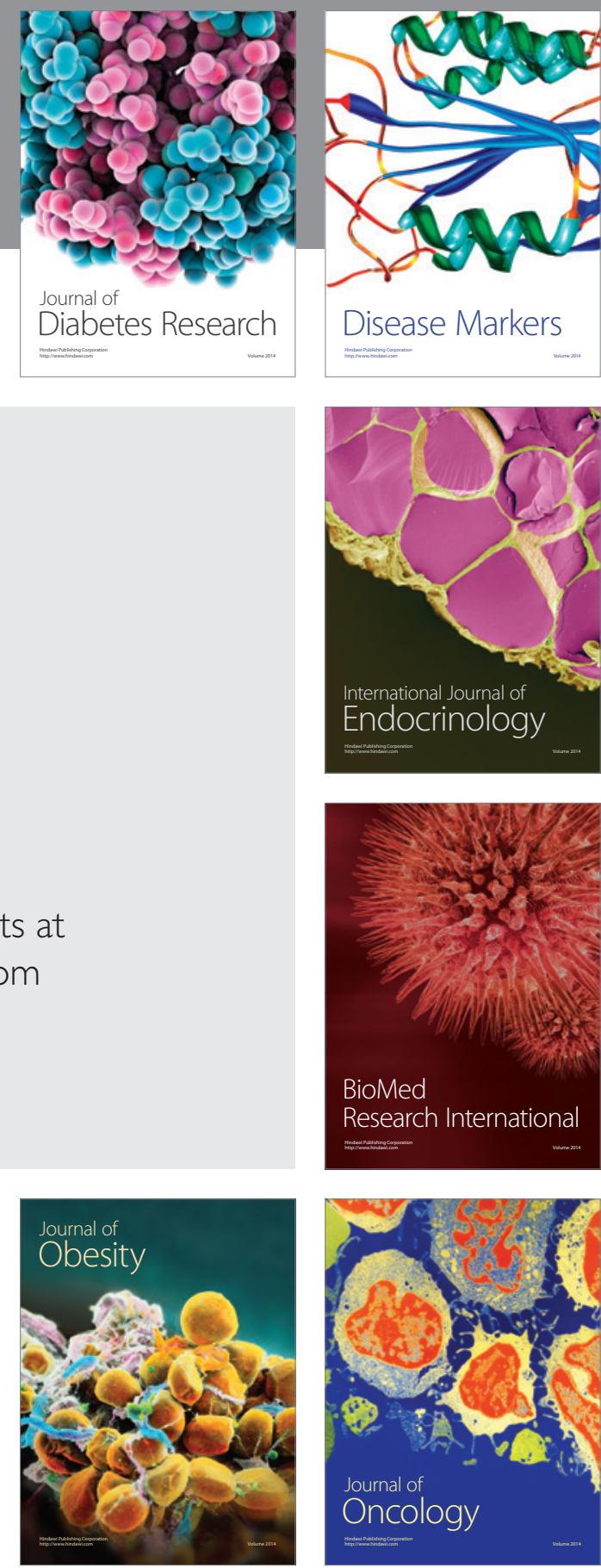

Disease Markers
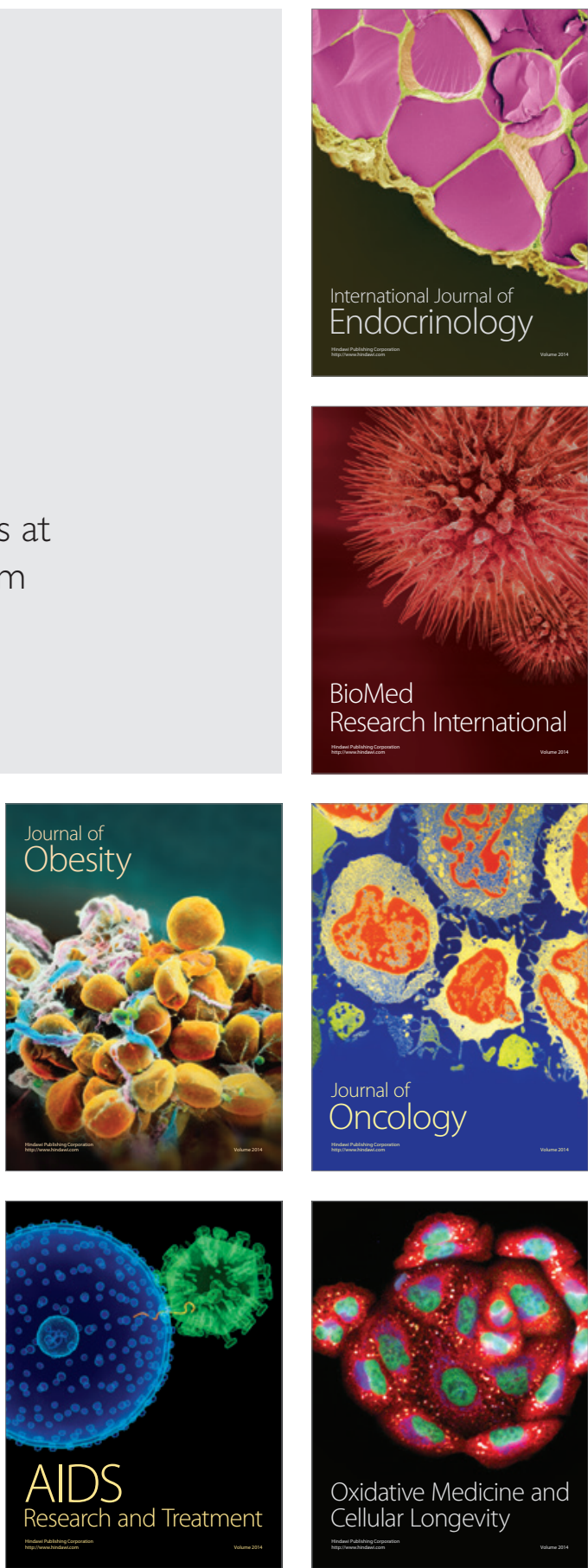